\title{
Paul Weingartner
}

\section{THE NEED FOR PLURALISM OF CAUSALITY}

\begin{abstract}
It will be shown in this article that a pluralism of causality is needed. Not only, as might be expected, for such different domains as natural sciences and humanities, but even within the domain of physics different causal relations are necessary. This will be illustrated with examples from Classical Mechanics and Special Relativity, Thermodynamics and Quantum Mechanics. In these domains causal relations differ in their properties. A model for five types of causal relations is provided that is based on a decidable 6 -valued logic.
\end{abstract}

Keywords: causality; causal explanation; causal sets; causal factors; necessary causes; sufficient causes

\section{Introduction}

The theory of causality by Salmon and Dowe (D\&S) will be used as an example of a non-pluralistic theory. Necessary features of Causal Processes and Causal Relations according to D\&S:

1. A causal process is a World Line (see [6, p. 90] and [24, p. 298])

2. of an object (see [6, pp. 90, 91])

3. that possesses a Conserved Quantity (see [6, p. 90] and [24, pp. 299, $303,306])$.

4. This World Line is continuous, a Continuous Trajectory (see [6, pp. 90f, 147f] and [24, p. 298]).

5. This World Line is in Minkowski Space-Time, belonging to the Special Theory of Relativity (for short: SR) (see [6, p. 90] and [24, p. 298]).

6. Conditions 2 and 3 presuppose that the object has identity over time (see [6, pp. 91, 101f]). 
7. Statistical characterizations of causal processes or relations are inadvisable according to D\&S (see [5, pp. 204ff], [6, pp. 33f], [23, p. 174], and $[24$, p. 301]).

Features 1-7 are best satisfied by objects and causal processes of Classical Mechanics (CM). The condition of World Line fits Minkowski Space-Time and SR and CM-objects which possess always a well-defined position in space. Features 1, 4, 5, and 6 rule out causal connections in Quantum Mechanics (QM). Elementary particles do not have unique trajectories. There is Permutational Invariance, i.e., indistinguishability of QM-objects of the same kind. D\&S mislead by giving examples of QM, although their concept of causality cannot be applied to them. Continuity is not satisfied in QM $(h \cdot \nu-j u m p)$ as has been mentioned above.

Identity over time or reidentifiability is not satisfied in QM because of permutational invariance. The claim by D\&S that statistical characterizations are not advisable is in conflict with their using examples of decay phenomena.

D\&S causality is an important type of causality but restricted to CM and SR. Another non-pluralistic and narrow approach to causality is due to Kiczuk [13]. Such non-pluralistic approaches require the investigation and incorporation of other types of causes and causal relations.

\section{Three Main Types of Causes}

\subsection{Causes as Causal Factors}

In most cases of everyday life and of science causes are "Causal Factors" $(C F)$ which are neither necessary nor sufficient.

Examples (Causal Factors).

- Amazon has hundreds of tributary rivers. A change of the temperature of one of them is a causal factor for the temperature of Amazon.

- Self-control in early childhood seems to lead to more health, more stable financial status and less criminality [18]. It is a $C F$.

Observe that causal factors can be statistically highly significant, it can have strong statistical relevance for the effect (cf. [27, p. 106]). For example: Some of the tributary rivers of Amazon can be, according to long-term statistics, highly significant for its temperature. Thus Rio 
Negro, which joins Amazon in Manaus coming from the north, is $7-10^{\circ} \mathrm{C}$ warmer when joining. The long term study of Moffitt et al. (more than 40 years with the same sample of test persons) seems to show that self control is very significant. Both examples indicate that causal factors can come close to causes as sufficient for the effect (sufficient causes). A causal factor may also come close to a cause as a necessary condition. This is the ease when the causal factor is a very significant and necessary element in a set of together sufficient conditions.

Properties of $C F$ : irreflexive, asymmetric or not-symmetric, nottransitive, continuous or discontinuous.

\subsection{Causes as Sufficient Conditions $(C S)$}

The famous historical example is Leibniz with his Principle of Sufficient Reason:

It is certain, therefore, that all truths, even the most contingent, have an a priori proof, or some reason why they are rather than are not. And this is itself what people commonly say, that nothing happens without a cause, or that nothing is without a reason.

$[15$, vol. 7 , p. 300]

Examples (Sufficient Conditions - CS).

- The guillotine-stroke is a $C S$ for death.

- Events of the past light cone are $C S$ for the events in the future light cone in SR.

$C S$ in SR is irreflexive, asymmetric, transitive, continuous. This holds also in CM. In CM, the causal relation is completely observerinvariant. In SR, although time measurement, simultaneity and spacial distance of simultaneous events are not generally invariant under transformations of inertial systems, with the help of the Lorentz Transformation the causality relation can be kept observer-invariant.

To interpret the events of the past light cone as $C S$ is incomplete. In fact, only these events plus the law structure is sufficient for the future events. More accurately: The past events are $C F_{\mathrm{s}}$, the law structure (represented by the dynamical laws) is a cause as necessary condition $(C N)$ and $C F+C N$ are sufficient $(C S)$ for the effect.

This fact comes closer to the intuitive idea of causation by the Hamiltonian formulation of CM, since what happens at space-time point $x_{2} t_{2}$ is not "caused" by the earlier space-time point $x_{1} t_{1}$ alone, but also by the forces which act on the particle in question (cf. [17, p. 236]). 


\section{Examples.}

- A change of $V$ (volume, or $T$, temperature) leads to a change of $P$ (pressure).

- The change of the length of a spherical pendulum leads to a change of the time of oscillation.

$C S$ is irreflexive, asymmetric, continuous. Also here the respective changes are only $C F$ s. Only together with the respective law they are sufficient.

Observe: Physically we cannot change the time of oscillation directly, but only the length; thus changing the length is a $C F$. Mathematically one can change any magnitude with the consequence of changing others. This leads to the following important distinction: The law of the spherical pendulum or the general gas law can be understood in two ways:

(a) As equations representing mathematical relations among magnitudes. Then, to (numerically) alter one magnitude leads via the equation to an (numerical) alteration of another magnitude. Any magnitude can be so altered, in the above cases: length, period of oscillation or pressure, temperature, volume. This has nothing to do with causality. There is no causality in mathematics except in the symbolic sense that premises may be called the causes for the conclusion which would also fit to logic.

(b) As physically interpreted equations representing physical laws corresponding ontologically to a structure of nature. Then, to change the length of a pendulum (i.e., of a physical material system) leads via the law (expressed by the equation) to a change of the period of oscillation of this pendulum (real physical material system). It is not possible to do it the other way round, i.e., to change the period of oscillation with the consequence of a change of the length. Therefore this is a good reason to call the change of the length a causal factor $(C F)$. These types of asymmetries have been called explanatory asymmetries by Hausman $[9$, pp. 158ff]. In general, mathematical changes in a mathematical equation are not always possible as physical changes, if this equation has a physical interpretation. This important distinction seems to have been recognized clearly by Karl Pearson, who distinguishes the wider concept of correlation from causation and interprets the relation of cause to effect as depending on contingent facts (cf. [20, pp. 339ff]).

Keeping this distinction in mind avoids a lot of irrelevant difficulties. The distinction can also be applied to the DN-Model of scientific expla- 
nation (due to Hempel and Oppenheim). Just replace - per analogiam the mathematical equation by the logical argument-structure of the DNModel: Well formed formulas as instances of the DN-Model and their changes, in such a way that the logical validity is preserved, do not in general lead to a scientific explanation. Why should one think that a combination of Modus Ponens + Universal Instantiation would produce a scientific explanation? Even if we agree that scientific explanations often have that structure, one cannot conclude the opposite that this logical structure leads to a scientific explanation. Many of the "counterexamples" to the DN-Model (cf. [36, pp. 154ff]) seem to be based on this logical fallacy.

Examples.

- A photon causes an electron to jump up from the ground state to the first excited state.

- The light-electric effect (Einstein 1905).

- $\alpha$-decays produce liquid droplets in the cloud chamber.

The photons and the $\alpha$-particles are CFs. Only together with the laws of QM of electromagnetism (EM) and of decay (DC) they are sufficient causes $(C S)$ for the effects.

Observe: Here are statistical laws involved (DC). Thus, the respective causal process is not describable by dynamical laws only. We have what Wolfgang Pauli called "statistical causality".

$C S$ and $C F$ are irreflexive, asymmetric, discontinuous, not transitive. The causal process is not a trajectory - except in the cloud chamber. In the causal model developed in Section 6, $C S$ and $C F$ are interpreted as discontinuous satisfying causal sets.

Examples.

- The entropy $E\left(M_{1} t_{1}\right)$ of a macrostate $M_{1}$ at $t_{1}$ develops into the greater entropy $E^{\prime}\left(M_{2} t_{2}\right)$.

- The DNA-polymerase III reduplicates the DNA.

- A certain mutation $m$ of the DNA causes a certain illness.

$E\left(M_{1} t_{1}\right)$, DNA-polymerase III, and mutation $m$ are $C F$ s for the respective effects.

\subsection{Causes as Necessary Conditions $(C N)$}

Necessary Conditions $(C N)$ has a longer tradition than Sufficient Conditions $(C S)$ : 
- Aristotle: His causa materialis and his causa formalis are CN. [1, ch. 2].

- Thomas Aquinas: "To take away the cause is to take away the effect" $[29, \mathrm{I}, 2,3]$.

- David Hume: "We may define a cause to be an object followed by another [...] where if the first object had not been, the second has never existed" [11, VII, 2].

Reasons for Aquinas to take cause as $C N$ in his Five Ways: If God were a cause (for the world) as $C S$, then:

(i) no cooperation of creatures as "secondary causes" (for example: no evolution) is possible;

(ii) there is no possibility for learning of creatures by trial and error;

(iii) God would cause every evil including moral evil, i.e., being inconsistent by giving the Ten Commandments.

Therefore, Aquinas interprets God as a necessary cause for his creation, but not as a sufficient one. As Gödel says: "God created things in such a way that they themselves can create something" $[8,9]$.

Examples (Necessary Conditions).

- The grandparents are $C N$ for the grandchildren.

- "The observed values of all physical and cosmological quantities are not equally probable, but they take on values [...]" [2, p. 16] that are $C N$ for carbon-based life.

$C S$ is sometimes transitive, sometimes not (cf. 3.5 and 4.3). The causal process in Minkowski Space-Time is transitive. $C N$ is usually transitive. A particular event of the past light cone is not a $C N$, but some or other is. The change of length of the spherical pendulum is a $C N$. The change of either volume or temperature is a $C F$ for the change of pressure. The absorption of a photon is not a $C N$ for the excited state of the electron because it may be also caused by particle-collision; but some energy input is a $C N$. The strong electrostatic repulsion is a $C N$ for the $\alpha$-decay. In all the examples the laws - ontologically speaking the law structure of nature-are $C N \mathrm{~s}$ for the respective effect. $C N$ is irreflexive, asymmetric, continuous or discrete, and transitive in the cases where transitivity is applicable. In the causal model developed in Section 6, $C N$ is interpreted as discrete satisfying causal sets (cf. Section 6.4). 


\section{Properties of Causal Relations}

\subsection{Terminology and Semantics}

1. $p, q, r, \ldots, \neg p, p \vee q, p \wedge q, p \rightarrow q, p \leftrightarrow q$ are wellformed formulas (wffs) of Propositional Calculus. In truth-tables semantics of two-valued Classical Propositional Calculus (in short: CPC) any formula is represented by a certain truth-matrix which has $2^{n}$ valuations, where $n$ is the number of propositional variables in a formula.

2. The 6-valued basic logic RMQ (see Section 5) of the model RMQC (see Section 6) contains the wffs of CPC as a proper part. Moreover, its formulas are extended by a modal operator $L(M:=\neg L \neg)$. The model presented in Section 6 is a further extension of five additional compound wffs: $p \mathrm{CF} q, p \mathrm{CN} q, p \mathrm{CS}_{1} q, p \mathrm{CS}_{2} q, p \mathrm{CS}_{3} q$ (the last three are abbreviated as $p \mathrm{CS} q$ ). RMQ and RMQC contain all theses of $\mathrm{CPC}$ as a proper part.

3. Every formula is unambiguously represented by a certain particular truth-matrix which has $6^{n}$ valuations, where $n$ is the number of propositional variables in a formula.

The values constitute the set $\{1,2,3,4,5,6\}$. We distinguish three values $1,2,3$ as values of truth. So we put $\mathbf{T}:=\{1,2,3\}$. Three values $4,5,6$ are values of falsity. So we put $\mathbf{F}:=\{4,5,6\}$.

A formula is valid (resp. contra valid) iff all its values in its matrix belong to $\mathbf{T}$ (resp. to $\mathbf{F}$ ). A formula is contingent iff both some its value in its matrix belongs to $\mathbf{T}$ and some its value in its matrix belongs to $\mathbf{F}$. Finally, a formula is invalid iff it is not valid, i.e., iff some its value in its matrix belongs to $\mathbf{F}$, i.e., iff it is either contra valid or contingent.

4. Wffs are understood as referring (ontologically) to states of affairs. Wffs that are valid are understood as referring to facts. Facts are understood as obtaining states of affairs. One may distinguish even three levels: states of affairs, facts and real facts (as defined in [30, ch. 8]). Wffs can refer to real facts only if they are true (valid), without negations, without disjunctions and satisfying relevance in the sense of $\mathrm{RC}$ and RD (see Section 5.2 and [30, ch. 9]). It holds between the three levels that every real fact is a fact and every fact is a state of affairs. States of affairs, facts or real facts are neither valid nor invalid only the wffs which represent them are.

5. States of affairs can be of different kinds: logical, mathematical, contingent, empirical causal. Thus $p \rightarrow p,(p \wedge q) \rightarrow p$ refer to logical states of affairs. 
Notice that every propositional variable refers to an contingent state of affairs. A formula containing three propositional variables has a matrix of 216 values (valuations), since $6^{3}=216$. This refers to a mathematical state of affairs.

Moreover, $p$ CS $q \rightarrow(p \rightarrow q)$ refers to empirical states of affairs. Finally, $p \mathrm{CF} q, p \mathrm{CN} q, p \mathrm{CS} q,(p \mathrm{CF} q \wedge p \mathrm{CN} q) \mathrm{CS} q$ refer to contingent causal states of affairs.

In the following sections these points $1-5$ are presupposed. However, we shall not explicitly write down "... refers to _ _ ", but write in the usual abbreviated way as in everyday language and in scientific discourse. So, for example, "event $p$ is a causal factor for event $q$ " is an abbreviation for "an event referred to by $p$ is a causal factor for an event referred to by $q$ ", which we write down as $p$ CF $q$.

The applied solution allows also for the use of the quantifier notation which simulates the use of formulas of propositional logic with quantifiers. For example, sentences of the form "For each event $p$ we have $A(p)$ " and "For all events $p$ and $q$ we have $A(p, q)$ " are abbreviations for "For every event referred to by $p$ it is such that $A(p)$ " and "For every event referred to by $p$ and for every event referred to by $q$ it is such that $A(p, q)$ ", which we write down as $\forall p A(p)$ and $\forall p \forall q A(p, q)$, respectively. Similarly, cases in which one or two 'every' are replaced by 'some', we write down as $\exists p A(p), \exists p \exists q A(p, q), \forall p \exists q A(p, q), \exists p \forall q A(p, q)$, respectively.

\subsection{Logical Relations}

We want to stress first that the material implication $p \rightarrow q$ does not say anything about a causal relation between $p$ and $q$; it does not involve time or a time-order either. The same holds for the strict implication $p \Rightarrow q$, where $p \Rightarrow q:=\mathrm{L}(p \rightarrow q)$. So no causal relation follows from material or strict implications.

However, if $p$ is a sufficient cause for $q$ (in short: $p$ CS $q$ ), then $p$ is a sufficient condition of $q$, i.e., the following formula is valid:

$$
p \mathrm{CS} q \rightarrow(p \rightarrow q)
$$

Analogously, if $p$ is a necessary cause for $q$ (in short: $p \mathrm{CN} q$ ), then $p$ is a necessary condition of $q$, i.e., the following formula is valid:

$$
p \mathrm{CN} q \rightarrow(q \rightarrow p)
$$


To be a causal factor, i.e. $p$ CF $q$, is weaker and does not imply either of the two. However, $p$ CF $q$ must be implied by both, i.e. we have:

$$
\begin{aligned}
p \mathrm{CS} q & \rightarrow p \mathrm{CF} q \\
p \mathrm{CN} q & \rightarrow p \mathrm{CF} q
\end{aligned}
$$

This is so as long as the causal factor is neither significant in the direction of a sufficient cause or in the direction of a necessary cause. In such cases the specific CF would be only implied by either CS or CN.

Let $C$ be either $\mathrm{CS}$, or $\mathrm{CN}$, or CF. We say that $C$ is irreflexive iff for every $p$ it is not the case that $p C p$. In the such case the following formula is valid:

$$
\neg p C p
$$

$C$ is transitive iff for all $p, q, r$ : if $p C q$ and $q C r$, then $p C r$. In such case the following formula is valid:

$$
(p C q \wedge q C r) \rightarrow p C r
$$

$C$ is symmetrical iff for all $p, q$ : if $p C q$, then $q C p$. In such case the following formula is valid:

$$
p C q \rightarrow q C p
$$

Thus, $C$ is not symmetrical iff there are $p, q$ such that $p C q$, but $\neg q C p$. Moreover, $C$ is not transitive iff there are $p, q$, and $r$ such that $p C q$ and $q C r$, but $\neg p C r$. In such cases we will use, respectively, the following informal writing:

$$
\begin{gathered}
p C q \not \rightarrow q C p \\
(p C q \wedge q C r) \not \rightarrow p C r
\end{gathered}
$$

which means that the formulas (sym) and (tr) are invalid.

Finally, $C$ is asymmetrical iff there are no $p$ and $q$ such that both $p C q$ and $q C p$. In such case the following formula is valid:

$$
p C q \rightarrow \neg q C p
$$

\subsection{Continuity and Discontinuity}

Let $C$ be either CS, or CN, or CF. An Alexandroff set of a causal relation $p C q$ is the set of states (states of affairs, events) $r, r_{1}, r_{2}, \ldots, r_{n}$ between $p$ and $q$ such that $r_{i}$ is caused by its predecessor $p$ and causes its successor $q$; i.e., the set $\{r: p C r \wedge r C q\}$. We say that $p C q$ is continuous iff the elements of its Alexandroff sets can be mapped on real numbers. 
$p C q$ is discontinuous iff its Alexandroff set is finite. Moreover, $p C q$ is strongly discontinuous (discrete) iff there is no $r$ between $p$ and $q$, i.e., iff its Alexandroff set is empty. Finally, we say that $C$ is continuous (resp. discontinuous, strongly discontinuous) iff for all $p$ and $q: p C q$ is continuous (resp. discontinuous, strongly discontinuous).

Notice that CS, CN, and CF in Minkowski Space-Time (SR) are continuous. $\mathrm{CS}, \mathrm{CN}$, and $\mathrm{CF}$ in the photoelectric effect or in a quantum jump are strongly discontinuous. $\mathrm{CN}$ and $\mathrm{CF}$ in the genealogical tree are discontinuous. In all stochastic phenomena described by statistical laws like radiation, thermodynamic processes, osmoses, diffusion, electric transport, entropic processes, reduplication, mutation - presupposing that the ensembles are huge but finite $-\mathrm{CS}, \mathrm{CN}$, and $\mathrm{CF}$ are discontinuous.

\subsection{Temporal Order}

That the cause must be earlier than the effect does not have a straight forward tradition. Aristotle accepts it for the most frequent cases in which the cause is in potency w.r.t. the effect, but claims simultaneity if the cause is in actuality $[1, \mathrm{~V}, 2]$. Thomas Aquinas assumes generally that the cause must be earlier than the effect and uses this assumption to show the irreflexivity of the causal relation, since the cause cannot be prior to itself [29, I, 2, 3]. Newton interprets causes as his forces and claims simultaneity with the effect [19, definitions]. Kant seems to have thought that the cause must be earlier than the effect although the temporal order is not observable according to him [12, B233].

From SR (and GR) we know that

(i) every causal propagation needs time, and

(ii) the causal propagation has an upper speed limit, i.e., $c$, the velocity of light in vacuum.

These two conditions hold for CS, CN and CF.

An important assumption about time which does not follow from physical laws or accepted axioms is this: The time coordinate is not closed; although the space coordinates are. There are no closed timelike curves. This is called the chronology condition of space time (cf. [10, p. 189] and [17, p. 219]). This is essential for a realistic causality. Otherwise time-travel is possible. In the model of causal relations presented in Section 6 below, the chronology condition is satisfied for the causal relations $\mathrm{CN}, \mathrm{CS}_{2}, \mathrm{CS}_{3}$, defined there, because they are non-circular 
(asymmetric). The remaining causal relations $\mathrm{CF}$ and $\mathrm{CS}_{1}$ are at least not-symmetric and irreflexive (cf. Section 6.4).

\subsection{Transitivity}

Pearl proposed the following test for transitivity: is there a case as follows: state (event) $A$ is capable of changing state (event) $B$ and state (event) $B$ is capable of changing state (event) $C$; yet state (event) $A$ is incapable of changing state (event) $C$ (see [20, p. 237]). Applying this test to CS shows that CS is not generally transitive.

Examples.

- Car $A$ bumps (= makes a damage on the backside of) car $B$ and car $B$ bumps car $C$; yet car $A$ does not bump car $C$.

- By sending electromagnetic high grade energy (i.e., entropy is low), the sun $(A)$ causes $(\mathrm{CS})$ order and information on earth $(B)$, and by passing it through, $B$ causes (CS) low grade energy $(C)$ (distributing it to the environment); yet $A$ does not cause (CS) $C$. On the contrary, $A$ caused (CS) order and information, but not its opposite.

Interpreting cause as $\mathrm{CN}$, however, shows that $\mathrm{CN}$ is transitive. Conjectured result: $\mathrm{CN}$ is transitive, but $\mathrm{CS}$ is not in general transitive. So, respectively, we have (see Section 3.2):

$$
\begin{aligned}
&(p \mathrm{CN} q \wedge q \mathrm{CN} r) \rightarrow p \mathrm{CN} r \\
&(p \mathrm{CS} q \wedge q \mathrm{CS} r) \not \rightarrow p \mathrm{CS} r
\end{aligned}
$$

In the model presented in Section 6, $\mathrm{CN}$ is transitive and there are three relations of CS: one is transitive, the others are not transitive.

\subsection{Objectivity of the Causal Relation}

We say that a causality relation is objective iff it is observer-invariant, i.e., if it holds for any observer.

1. In CM, because of the assumption of universal time and simultaneity the causal relation is objective.

2. In SR the Lorentz transformation corrects the underlying (wrong) preconditions of Galilean invariance: that time measurement, simultaneity and spacial distance of simultaneous events are generally invariant w.r.t. inertial systems. By this correction the causality relation can be kept objective in SR. 
3. In GR the causality relation is only locally objective in regions where there is a light-cone structure.

4. In QM there are two restrictions of causality:

(a) the incomplete causality of the Schrödinger dynamics is applicable only for the subset of commensurable properties;

(b) the causality of the measurement process is complete but only statistically applicable, i.e., not relevant in single cases [17, ch. 9.3].

5. In Thermodynamics causality is twofold:

(a) on the macro-level, independently of an underlying micro-structure it is similar as in (1);

(b) on the micro-level as an explanatory causal structure of the macro-level it is statistical causality as in $4 \mathrm{~b}$ above.

\section{Causality Relations in Causal Explanations}

\subsection{Dynamical Laws}

A dynamical law describes the time development of a (physical, chemical, biological, psychological) system $\boldsymbol{S}$ in such a way that:

1. state $S_{2}$ at $t_{2}$ of $\boldsymbol{S}$ is a definite function (described by a differential equation) of earlier state $S_{1}$ at $t_{1}$ of $\boldsymbol{S}$.

2. The first condition 1 holds also for every part of $\boldsymbol{S}$.

3. There is a hidden assumption: the system $\boldsymbol{S}$ has a certain type of stability in the following sense: small changes in the initial states lead to proportionally small changes in the final states. This hidden assumption precludes chaotic behaviour. For CL this was a hidden assumption, since experiments showing dynamical chaos began only in 1984 (cf. [35, p. 51].

The causal structure of an explanation with dynamical laws looks as follows:

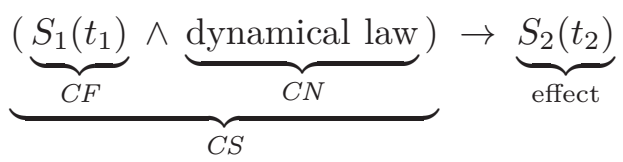

\subsection{Correlation Laws}

In Thermodynamics one studies the question, how the properties of a (physical, chemical, biological) system change with temperature. This can be studied in a twofold way:

(a) Only on the macro-level studying solely the relations between macroscopic observable quantities independent of an underlying struc- 
ture. To this domain pertain the so called "correlation laws" and the subsequent causal explanation.

(b) On the micro-level with the focus to use the behaviour on the micro-level as the explanatory and causal structure of the behaviour on the macro-level. This was developed in two ways, as the Kinetic Theory of Gases (by Clausius and Maxwell) and as the Statistical Mechanics (by Boltzmann and Gibbs). Causality on this level concerns the law of entropy (see Section 4.3) and laws of Quantum Mechanics. In a causal explanation on the level (a) the causal relation CS is irreflexive and continuous, but neither symmetric nor transitive (cf. Section 6.5.2).

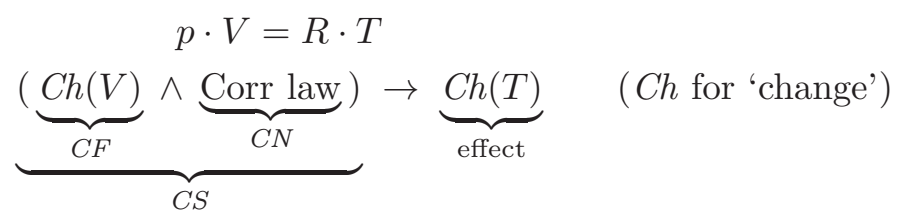

\subsection{Law of Entropy}

$E(M)=$ the finite (but huge) number of micro-states $(m)$, which can realize the macrostate $(M)$. The entropy $E$ of $M, E(M)$, of an (isolated) system $\boldsymbol{S}$ at time $t_{1}$ develops according to the law of entropy (LE) into a higher entropy $E^{\prime}(M)$ of $\boldsymbol{S}$ at $t_{2}$.

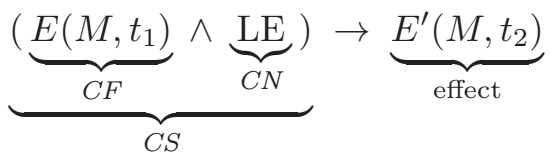

Concerning properties of the causal relations, they are also irreflexive and asymmetric. But here they are not continuous, but discrete. Since the law of entropy (LE) is a statistical law, we may speak of statistical causality. The transitivity of CS can be shown to be satisfied thus:

1. $E\left(M, t_{1}\right) \wedge L E \stackrel{\mathrm{CS}}{\longrightarrow} E\left(M, t_{2}\right) \wedge L E$

2. $E\left(M, t_{2}\right) \wedge L E \stackrel{\mathrm{CS}}{\longrightarrow} E\left(M, t_{3}\right)$

3. $E\left(M, t_{1}\right) \wedge L E \stackrel{\mathrm{CS}}{\longrightarrow} E\left(M, t_{3}\right)$

\subsection{Quantum Jump}

The input of energy $h \cdot \nu$ by absorption of a photon causes an electron to jump up from the ground state to the first excited state. 


$$
\begin{aligned}
& S_{0}(e l) \quad \ldots \quad \text { ground state of electron } \\
& S_{1}(e l) \quad \ldots \quad \text { first excited state of electron } \\
& E n g(p h) \quad \ldots \quad \text { energy input of a photon } \\
& \underbrace{(\underbrace{S_{0}(e l)}_{C N} \wedge \underbrace{\operatorname{Eng}(p h)}_{C F} \wedge \underbrace{\operatorname{law}(\mathrm{QM})}_{\mathrm{CN}})}_{C S} \rightarrow \underbrace{S_{1}(e l)}_{\text {effect }}
\end{aligned}
$$

Observe that the ground state $S_{0}(e l)$ is only a relative necessary cause if the final state is the first excited state. Because the jump can occur on higher levels $n, n+1$; the electron can even jump two levels at once depending on the energy input. The energy input of a photon is not a necessary cause since the jump of the electron can also be caused by particle collision. The causal relation is irreflexive, asymmetric, discrete and not transitive (cf. Section 6.5.4).

\subsection{Causality in the Cloud Chamber}

It is an experimental fact that $\alpha$-decays (Helium nuclei, 2 protons plus 2 neutrons) produce in a cloud chamber at most one track, i.e., a sequence of liquid droplets; this track points in a random direction. Decay phenomena cannot be described by dynamical laws. They are adequately describable by statistical laws. However, the single track is compatible with the trajectory of a classical particle with the same mass, energy and charge as the $\alpha$-wave produced in the decay. On the other hand, the decay should produce - according to Quantum Mechanics - a spherical wave that moves radially. This conflict in the explanation of the interaction of the emittel wave with the atoms in the cloud chamber is not a really solved problem so far (cf. [4] for recent discussion). We consider the two types of causal relations involved here (cf. Section 6.5.5):

1. The decay, i.e., the emission of $\alpha$-particles, is caused by electrostatic repulsion of the protons in heavy nuclei;

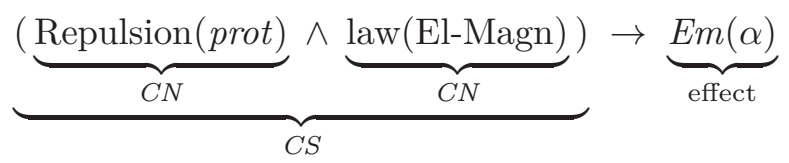

2. The production of liquid droplets is caused by the charged $\alpha$-particle interacting with atoms of the supersaturated vapour. 


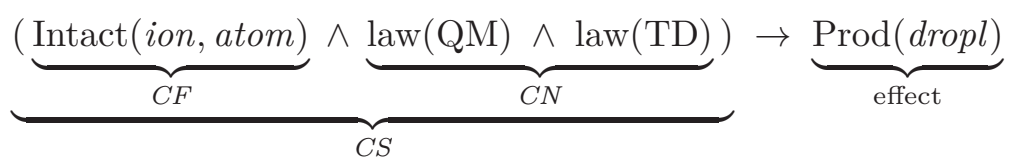

\section{The Basic Logic RMQ for the Model of Causal Relations}

The basic Logic for the model of causal relations is a propositional logic (see Section 3.1). This logic, which we call RMQ, is the set of all valid formulas in some six-values matrix (see [33]).

The basic logic RMQ has the following properties:

(1) RMQ is a 6-valued matrix system (3 values for truth, 3 for falsity) and so it contains its own semantics. Every well formed formula of RMQ is unambiguously determined by a particular matrix which contains $6^{n}$ values for $n(n=1,2, \ldots)$ different propositional variables. So RMQ is consistent and decidable, and RMQ has the finite model property.

(2) RMQ has two concepts of validity: a weaker one (materially valid) and a stronger one (strictly valid). All theorems of two-valued Classical Logic (Classical Propositional Calculus; CPC) are at least materially valid in RMQ. Only a restricted class of them are strictly valid in RMQ.

(3) The validity of a proposition is decided by calculating the highest value $(c v)$ in its particular matrix. If $c v(A)=3$, then the proposition (formula) $A$ is materially valid. If $c v(A) \leqslant 2$, then $A$ is strictly valid.

(4) The strictly valid theorems of RMQ avoid the well known difficulties when logic is applied to physics; especially those with commensurability, distributivity and with Bell's inequalities. They also avoid paradoxes in other domains, like theory of explanation, confirmation, verisimilitude, deontic logic.

(5) RMQ contains a modal system with 14 modalities.

First, this basic logic RMQ will be defined in Section 5.1 and two types of theorems (materially and strictly valid) will be presented (see sections 5.2 and 5.3). Second, it will be extended by the non-logical causal relations $\mathrm{CF}, \mathrm{CN}, \mathrm{CS}_{1}, \mathrm{CS}_{2}$ and $\mathrm{CS}_{3}$ (see Section 6).

\subsection{Definition of the basic logic RMQ}

The matrix for the basic logic RMQ we take from [33, p. 140]. This logic can be defined as the set of all formulas (wffs) which are valid in the matrix $\mathfrak{M}=\langle\mathbf{T}, \mathbf{F}, \neg, \vee, \wedge, \rightarrow, \mathrm{L}\rangle$, where $\mathbf{T}=\{1,2,3\}, \mathbf{F}=\{4,5,6\}$ and the operations $\neg, \vee, \wedge, \rightarrow, \mathrm{L}$ are defined in Table 1. 


\begin{tabular}{|c|c|}
\hline$p$ & $\neg p$ \\
\hline 1 & 6 \\
2 & 5 \\
3 & 4 \\
4 & 3 \\
5 & 2 \\
6 & 1 \\
\hline
\end{tabular}

\begin{tabular}{|c|cccccc|}
\hline$p \vee q$ & 1 & 2 & 3 & 4 & 5 & 6 \\
\hline 1 & 1 & 1 & 1 & 1 & 1 & 1 \\
2 & 1 & 2 & 2 & 2 & 1 & 2 \\
3 & 1 & 2 & 3 & 1 & 3 & 3 \\
4 & 1 & 2 & 1 & 4 & 4 & 5 \\
5 & 1 & 1 & 3 & 4 & 5 & 5 \\
6 & 1 & 2 & 3 & 5 & 5 & 6 \\
\hline
\end{tabular}

\begin{tabular}{|c|llllll|}
\hline$p \wedge q$ & 1 & 2 & 3 & 4 & 5 & 6 \\
\hline 1 & 1 & 2 & 3 & 4 & 5 & 6 \\
2 & 2 & 2 & 3 & 4 & 6 & 6 \\
3 & 3 & 3 & 3 & 6 & 5 & 6 \\
4 & 4 & 4 & 6 & 4 & 5 & 6 \\
5 & 5 & 6 & 5 & 5 & 5 & 6 \\
6 & 6 & 6 & 6 & 6 & 6 & 6 \\
\hline
\end{tabular}

\begin{tabular}{|c|c|cccccc|}
\hline$p \rightarrow q$ & 1 & 2 & 3 & 4 & 5 & 6 \\
\hline 1 & 1 & 2 & 3 & 5 & 5 & 6 \\
2 & 1 & 1 & 3 & 5 & 5 & 5 \\
3 & 1 & 2 & 1 & 4 & 5 & 5 \\
4 & 1 & 2 & 3 & 1 & 3 & 3 \\
5 & 1 & 2 & 2 & 2 & 1 & 2 \\
6 & 1 & 1 & 1 & 1 & 1 & 1 \\
\hline
\end{tabular}

\begin{tabular}{|c|c|}
\hline$p$ & $\mathrm{~L} p$ \\
\hline 1 & 1 \\
2 & 3 \\
3 & 6 \\
4 & 6 \\
5 & 6 \\
6 & 6 \\
\hline
\end{tabular}

Table 1. The truth-tables for $\neg, \wedge, \vee, \rightarrow$, and $\mathrm{L}$

In detail we may explain this as follows: We have constructed the matrix $\mathfrak{M}_{\mathrm{RMQ}}:=\langle\mathbf{V}, \mathbf{T}, \mathcal{C}\rangle$, where $\mathbf{V}:=\{1, \ldots, 6\}$ is the set of the considered values, $\mathbf{T}:=\{1,2,3\}$ is the set of designated values, and $\mathcal{C}$ is the set of functions that are interpretations of propositional connectives $\neg, \wedge, \vee, \rightarrow$, and $L$ that are defined by the truth-tables from Table 1 . So three values from $\mathbf{T}$ we distinguish for truth. Three values 4, 5, 6 are for falsity. So we put $\mathbf{F}:=\{4,5,6\}$.

By using the truth-tables from Table 1 for each formula of RMQ we can construct its particular matrix. If a formula has $n$ propositional variables, then its matrix has $6^{n}$ valuations. Formally, a value of a formula $A$ in its particular matrix is equal to $h(A)$ for some homomorphisms $h$ from the set of formulas $\mathcal{F}$ into $\mathbf{V}$ for $\mathfrak{M}_{\mathrm{RMQ}}$.

Notice that the connectives $\neg, \vee, \wedge$, and $\rightarrow$ satisfy classical conditions, i.e., for all $h \in \operatorname{hom}(\mathcal{F}, \mathbf{V})$ and $A, B \in \mathcal{F}$ we have:

$$
\begin{aligned}
h(\neg A) & \in \mathbf{T} \text { iff } h(A) \in \mathbf{F}, \\
h(A \vee B) & \in \mathbf{T} \text { iff either } h(A) \in \mathbf{T} \text { or } h(B) \in \mathbf{T}, \\
h(A \wedge B) & \in \mathbf{T} \text { iff both } h(A) \in \mathbf{T} \text { and } h(B) \in \mathbf{T}, \\
h(A \rightarrow B) & \in \mathbf{T} \text { iff either } h(A) \in \mathbf{F} \text { or } h(B) \in \mathbf{T} .
\end{aligned}
$$

We will use material equivalence $\leftrightarrow$ as the following abbreviation:

$$
A \leftrightarrow B:=(A \rightarrow B) \wedge(B \rightarrow A)
$$




\begin{tabular}{|c|llllll|}
\hline$p \leftrightarrow q$ & 1 & 2 & 3 & 4 & 5 & 6 \\
\hline 1 & 1 & 2 & 3 & 5 & 5 & 6 \\
2 & 2 & 1 & 3 & 6 & 6 & 5 \\
3 & 3 & 3 & 1 & 6 & 6 & 5 \\
4 & 5 & 6 & 6 & 1 & 3 & 3 \\
5 & 5 & 6 & 6 & 3 & 1 & 2 \\
6 & 6 & 5 & 5 & 3 & 2 & 1 \\
\hline
\end{tabular}

Table 2. The truth-table for $\leftrightarrow$

\begin{tabular}{|c|c|}
\hline$p$ & $\mathrm{M} p$ \\
\hline 1 & 1 \\
2 & 1 \\
3 & 1 \\
4 & 1 \\
5 & 4 \\
6 & 6 \\
\hline
\end{tabular}

\begin{tabular}{|c||c|c|c|c|c|c|c|}
\hline$p$ & $\mathrm{~L} \mathrm{~L} p$ & $\mathrm{~L} p$ & $\mathrm{M} \mathrm{L} p$ & $p$ & $\mathrm{~L} M p$ & $\mathrm{M} p$ & $\mathrm{M} \mathrm{M} p$ \\
\hline 1 & 1 & 1 & 1 & 1 & 1 & 1 & 1 \\
2 & 6 & 3 & 1 & 2 & 1 & 1 & 1 \\
3 & 6 & 6 & 6 & 3 & 1 & 1 & 1 \\
4 & 6 & 6 & 6 & 4 & 1 & 1 & 1 \\
5 & 6 & 6 & 6 & 5 & 6 & 4 & 1 \\
6 & 6 & 6 & 6 & 6 & 6 & 6 & 6 \\
\hline
\end{tabular}

Table 3. The truth-tables for $M$ and for all positive modalities in RMQ

Notice that, by (5.7) and (5.8), also $\leftrightarrow$ satisfies a classical condition, i.e., for all $h \in \operatorname{hom}(\mathcal{F}, \mathbf{V}) A, B \in \mathcal{F}$ we have (see Table 2):

$$
h(A \leftrightarrow B) \in \mathbf{T} \text { iff either } h(A), h(B) \in \mathbf{T} \text { or } h(A), h(B) \in \mathbf{F} .
$$

Moreover, for L we obtain:

$$
\begin{aligned}
& h(\mathrm{~L} A) \in \mathbf{T} \text { iff } h(A) \in\{1,2\}, \\
& h(\mathrm{~L}(A \wedge B))=h(\mathrm{~L} A \wedge \mathrm{L} B) .
\end{aligned}
$$

So the formulas $\mathrm{L}(p \wedge q)$ and $\mathrm{L} q \wedge \mathrm{L} q$ have the same values in their particular matrixes, i.e., the highest value in the particular matrix of $\mathrm{L}(p \wedge q) \leftrightarrow(\mathrm{L} q \wedge \mathrm{L} q)$ is equal to 1 .

The logic RMQ obeys the usual interdefinability between necessity $\mathrm{L}$ and possibility $M$, i.e., we will use the following abbreviation:

$$
\mathrm{M} A:=\neg \mathrm{L} \neg A
$$

The logic RMQ has 14 modalities. In Table 3 we give the truth-tables for $M$ and for seven positive modalities in RMQ.

Moreover, for strict implication $\Rightarrow$ and strict equivalence $\Leftrightarrow$ we use the following abbreviations:

$$
\begin{aligned}
& A \Rightarrow B:=\mathrm{L}(A \rightarrow B) \\
& A \Leftrightarrow B:=\mathrm{L}(A \leftrightarrow B)
\end{aligned}
$$




\begin{tabular}{|c|cccccc|}
\hline$p \Rightarrow q$ & 1 & 2 & 3 & 4 & 5 & 6 \\
\hline 1 & 1 & 3 & 6 & 6 & 6 & 6 \\
2 & 1 & 1 & 6 & 6 & 6 & 6 \\
3 & 1 & 3 & 1 & 6 & 6 & 6 \\
4 & 1 & 3 & 6 & 1 & 6 & 6 \\
5 & 1 & 3 & 3 & 3 & 1 & 3 \\
6 & 1 & 1 & 1 & 1 & 1 & 1 \\
\hline
\end{tabular}

\begin{tabular}{|c|cccccc|}
\hline$p \Leftrightarrow q$ & 1 & 2 & 3 & 4 & 5 & 6 \\
\hline 1 & 1 & 3 & 6 & 6 & 6 & 6 \\
2 & 3 & 1 & 6 & 6 & 6 & 6 \\
3 & 6 & 6 & 1 & 6 & 6 & 6 \\
4 & 6 & 6 & 6 & 1 & 6 & 6 \\
5 & 6 & 6 & 6 & 6 & 1 & 3 \\
6 & 6 & 6 & 6 & 6 & 3 & 1 \\
\hline
\end{tabular}

Table 4. The truth-tables for $\Rightarrow$ and $\Leftrightarrow$

In Table 4 we give the truth-tables for $\Rightarrow$ and $\Leftrightarrow$. The truth-table for $\Rightarrow$ shows that for all $h \in \operatorname{hom}(\mathcal{F}, \mathbf{V})$ and $A, B \in \mathcal{F}$ :

$$
\text { if } h(A), h(A \Rightarrow B) \in \mathbf{T} \text {, then } h(B) \in \mathbf{T},
$$

Moreover, by (5.11), for all $h \in \operatorname{hom}(\mathcal{F}, \mathbf{V})$ and $A, B \in \mathcal{F}$ we have:

$$
h(A \Leftrightarrow B)=h(A \Rightarrow B \wedge B \Rightarrow A) .
$$

So the formulas $p \Leftrightarrow q$ and $(p \Rightarrow q \wedge q \Rightarrow p$ ) have the same values in their particular matrixes.

We say that a formula $A$ is valid in the matrix $\mathfrak{M}_{\mathrm{RMQ}}$ (or in the logic RMQ) iff for any $h \in \operatorname{hom}(\mathcal{F}, \mathbf{V})$ we have $h(A) \in \mathbf{T}$. Formulas which are valid in $\mathfrak{M}_{\mathrm{RMQ}}$ are theses of RMQ.

Notice that, by (5.5)-(5.9) and (5.12), we obtain:

- All theses of CPC and all their instances are valid in $\mathfrak{M}_{\mathrm{RMQ}}$.

- The set of all valid formulas in $\mathfrak{M}_{\mathrm{RMQ}}$ is closed under modus ponens for $\rightarrow$ and $\Rightarrow$, and it is closed under transitivity of $\rightarrow$, i.e., for all $A, B, C \in \mathcal{F}$ we have, respectively:

- if $A$ and $A \rightarrow B$ are valid in $\mathfrak{M}_{\mathrm{RMQ}}$, then so is $B$;

- if $A$ and $A \Rightarrow B$ are valid in $\mathfrak{M}_{\mathrm{RMQ}}$, then so is $B$;

- if $A \rightarrow B$ and $B \rightarrow C$ are valid in $\mathfrak{M}_{\mathrm{RMQ}}$, then so is $A \rightarrow C$.

The logic RMQ has two concepts of validity: a weaker one-materially valid formulas and a stronger one - then we say that given formulas are strictly valid.

We say that a formula $A$ is materially valid in RMQ iff $A$ is valid in $\mathfrak{M}_{\mathrm{RMQ}}$ but for some $h \in \operatorname{hom}(\mathcal{F}, \mathbf{V})$ we have $h(A)=3$, i.e., iff the highest value in the particular matrix of $A$ equals 3 (in short: $c v(A)=3$ ).

We say that a formula $A$ is strictly valid in the logic RMQ iff for any $h \in \operatorname{hom}(\mathcal{F}, \mathbf{V})$ we have $h(A) \in\{1,2\}$, i.e., iff in the particular matrix of $A$ we have $\operatorname{cv}(A) \leqslant 2$. 
Notice that, by (5.10), we have:

(i) a formula $A$ is strictly valid in RMQ iff $\mathrm{L} A$ is valid in $\mathfrak{M}_{\mathrm{RMQ}}$;

(ii) so $A \rightarrow B$ is strictly valid in RMQ iff $A \Rightarrow B$ is valid in $\mathfrak{M}_{\mathrm{RMQ}}$;

(iii) if $B$ is strictly valid in RMQ, then $A \Rightarrow B$ valid in $\mathfrak{M}_{\mathrm{RMQ}}$.

Now notice that the truth-tables for $\rightarrow$ and $\Rightarrow$ show that for all $h \in \operatorname{hom}(\mathcal{F}, \mathbf{V})$ and $A, B \in \mathcal{F}$ we have:

- if $h(A) \in\{1,2\}$ and $h(A \rightarrow B) \in\{1,2\}$, then $h(B) \in\{1,2\}$;

- if $h(A) \in\{1,2\}$ and $h(A \Rightarrow B)=1$, then $h(B) \in\{1,2\}$.

Hence we obtain:

- the set of all strictly valid formulas in RMQ is closed under modus ponens for $\rightarrow$ and $\Rightarrow$, i.e., for all $A, B \in \mathcal{F}$ we have, respectively:

- if $A$ and $A \rightarrow B$ are strictly valid, then so is $B$;

- if $A$ and $A \Rightarrow B$ are strictly valid, then so is $B$.

Moreover:

- the set of all materially valid formulas in RMQ is closed under modus ponens for $\rightarrow$, i.e., for all $A, B \in \mathcal{F}$ we have:

- if $A$ and $A \rightarrow B$ are materially valid, then so is $B$.

This follows from the following stronger condition which we obtain by (ii) and (iii):

- if $A$ is valid in $\mathfrak{M}_{\mathrm{RMQ}}$ and $A \rightarrow B$ is materially valid, then $B$ is also materially valid.

Notice that some theses of CPC are not strictly valid in RMQ (see the next Section 5.2).

Because of its two concepts of validity, RMQ separates those classically logical principles which usually lead to difficulties and paradoxes as materially valid from those which do not lead to difficulties as strictly valid. It is interesting to see that most of the well-known traditional logical principles like modus ponens, modus tollens, hypothetical syllogism, contraposition, disjunctive syllogism etc. are all strictly valid in RMQ. Accordingly, we shall list first some principles which are only materially valid (Section 5.2) and then some which are strictly valid (Section 5.3).

The strictly valid theorems of RMQ avoid the well known difficulties when logic is applied to physics; especially those with commensurability, distributivity and with Bell's inequalities. They also avoid paradoxes in other domains, like theory of explanation, confirmation, verisimilitude, deontic logic. 
The logic RMQ satisfies almost completely the two relevance criteria: $\mathrm{RC}$ - replacement; and RD - reduction (see below in Section 5.2). It has been shown in a number of publications that these two criteria can avoid difficulties and paradoxes in different domains, where Classical Logic is applied to empirical sciences (see [25, 26, 30, 31, 32, 34]).

\subsection{Some Relevance Criteria}

In [33, pp. $147 \mathrm{f}$ and 153f] it is shown that the following theses of CPC are not strictly valid in $\mathrm{RMQ},{ }^{1}$ since the highest value in their particular matrix equals $3(c v=3)$ :
1. $\neg p \rightarrow(p \rightarrow q)$
2. $\neg p \rightarrow[p \rightarrow(q \wedge \neg q)]$
3. $(p \rightarrow \neg p) \rightarrow[p \rightarrow(q \wedge \neg q)]$
4. $p \rightarrow(p \vee q)$
5. $p \rightarrow[p \vee(q \wedge \neg q)]$
6. $p \rightarrow[(p \wedge q) \vee(p \wedge \neg q)]$
7. $(p \rightarrow q) \rightarrow[(p \wedge r) \rightarrow(q \wedge r)]$
8. $(p \rightarrow q) \rightarrow[(p \vee r) \rightarrow(q \vee r)]$
9. $p \rightarrow(q \rightarrow p)$
10. $p \rightarrow[(q \vee \neg q) \rightarrow p]$
11. $p \rightarrow[q \rightarrow(p \wedge q)]$
12. $(p \wedge q) \rightarrow(p \leftrightarrow q)$
13. $(p \wedge q) \rightarrow(p \rightarrow q)$
14. $(p \wedge q) \rightarrow[(q \wedge \neg q) \vee(q \wedge p)]$
15. $(p \wedge q) \rightarrow[(p \wedge r) \vee(q \wedge \neg r)]$
16. $p \rightarrow[p \vee(p \wedge q)]$
17. $p \rightarrow[p \wedge(p \vee q)]$
18. $p \leftrightarrow[(p \wedge q) \vee(p \wedge \neg q)]$
19. $p \leftrightarrow[p \wedge(q \vee \neg q)]$
20. $p \vee(p \rightarrow q)$
21. $(p \rightarrow q) \vee(p \rightarrow \neg q)$
22. $(p \rightarrow q) \vee(\neg p \rightarrow q)$

ex falso quod libet ex falso quod libet ex falso quod libet redundant element(s) redundant element(s) redundant element(s) redundant element(s) redundant element(s) adding premise adding premise adding premise conjunction and equivalence conjunction and implication conjunction and disjunction conjunction and disjunction

A material implication $A \rightarrow B$ satisfies the replacement criterion (in short: RC) iff $A \rightarrow B$ is a thesis of CPC and it is not the case that some

1 Notice that all theses of $\mathrm{CPC}$ are valid in $\mathfrak{M}_{\mathrm{RMQ}}$ (i.e. they are theses of RMQ). 
propositional variable is replaceable in $B$ on some of its occurrences by any other propositional variable salva validate of $A \rightarrow B$ in CPC. ${ }^{2}$

It is easy to see that the above theses $1-19$ violate $\mathrm{RC}$. Indeed, $q$ (resp. $p$ or $r$ ) can be replaced in the consequent part on one or more than one occurrences salve validate of the respective thesis of CPC. ${ }^{3}$

Observe that equivalences have to be split up into the two implications in order to apply $\mathrm{RC}$. If one of the implications violates $\mathrm{RC}$ then also the equivalence violates $\mathrm{RC}$.

We say that $B$ is a relevant consequence of $A$ iff the material implication $A \rightarrow B$ satisfies the replacement criterion.

Observe that the thesis 15 is a propositional form of Bell's inequalities that is ruled out by $\mathrm{RC}$ and thrown out as not strictly valid in RMQ. The respective (ruled out) probabilistic forms and more details on restricted distributivity and commensurability are discussed in [33, pp. 148-153].

We say that $B$ is a reduced consequence of $A$ iff both $B$ is a relevant consequence of $A$ and $B$ is not logically equivalent to a conjunction $B_{1} \wedge \cdots \wedge B_{n}(n>0)$, in which $B_{i}$ is shorter than $B$, for $i=1, \ldots, n$.

Finally, we say that a material implication $A \rightarrow B$ satisfies the reduction criterion (in short: RD) iff $B$ is a reduced consequence of $A$.

The gist of the criterion to avoid reducible parts (reduction criterion) is as follows:

(a) it reduces repetitions;

(b) it reduces double negations;

(c) it splits complex wffs into smallest conjuncts;

(d) the process of splitting up is asymmetric in the sense that it leads from disjuncts to conjuncts (as the ultimate relevant consequence elements) but not back; however because RD splits up it does not allow building up arbitrarily conjunctions from separated parts.

The logic RMQ approximates very closely RC and RD (see [33]).

2 This criterion originates by Schurz and Weingartner in [25]. There and in some of the above mentioned papers it is more generally formulated for PL1 (including classical propositional calculus CPC, where propositional variables are interpreted as zero-type predicates).

3 To the theses 20-22 RC is not applicable, because they do not have the form of an implication. 


\subsection{Examples of Theses of CPC which are strictly valid in RMQ}

The following formulas are valid in $\mathfrak{M}_{\mathrm{RMQ}}$. Notice that, by definitions, $A \Rightarrow B$ (resp. $A \Leftrightarrow B$ ) is valid in $\mathfrak{M}_{\mathrm{RMQ}}$ iff $A \rightarrow B$ (resp. $A \leftrightarrow B$ ) is strictly valid in RMQ. Thus, all theses of CPC of the form $A \rightarrow B$ (resp. $A \leftrightarrow B$ ), which are strictly valid in RMQ, are recognisable by their main connective $\Rightarrow$ (resp. $\Leftrightarrow)$. For comparison also some theses of CPC, which are not strictly valid in RMQ (i.e. which are only materially valid), are listed below with $\rightarrow$. All the main thesis which hold strictly (i.e. with $\Rightarrow$ ), are valid in a logic suitable for QM.

1. $(p \wedge q) \Leftrightarrow(q \wedge p)$

commutation

2. $(p \vee q) \Leftrightarrow(q \vee p)$ commutation

3. $[p \wedge(q \wedge r)] \Leftrightarrow[(p \wedge q) \wedge r)]$ association

4. $p \Rightarrow p$

5. $p \Leftrightarrow \neg \neg p$

6. $p \wedge q \Rightarrow p$

7. $p \wedge q \Rightarrow q$

8. $p \vee p \Rightarrow p$

9. $[(p \rightarrow q) \wedge p] \Rightarrow q$

10. $[(p \rightarrow q) \wedge \neg q] \rightarrow \neg p$

11. $(p \rightarrow q) \Rightarrow(\neg q \rightarrow \neg p)$

12. $[(p \rightarrow q) \wedge(q \rightarrow r)] \Rightarrow(p \rightarrow r)$

double negation simplification simplification simplification modus ponens modus tollens contraposition

13. $[(p \vee q) \wedge \neg p] \Rightarrow q$

14. $(p \wedge q) \Rightarrow \neg(\neg p \vee \neg q)$

15. $(p \vee q) \Rightarrow \neg(\neg p \wedge \neg q)$

16. $(\neg p \wedge \neg q) \Rightarrow \neg(p \vee q)$

17. $(\neg p \vee \neg q) \Rightarrow \neg(p \wedge q)$

18. $\neg(\neg p \vee \neg q) \rightarrow(p \wedge q)$

19. $\neg(\neg p \wedge \neg q) \rightarrow(p \vee q)$

20. $\neg(p \vee q) \rightarrow(\neg p \wedge \neg q)$

21. $\neg(p \wedge q) \rightarrow(\neg p \vee \neg q)$

22. $[(p \wedge q) \vee(p \wedge r)] \Rightarrow[p \wedge(q \vee r)]$ hypothetic syllogism disjunctive syllogism de Morgan's law de Morgan's law de Morgan's law de Morgan's law de Morgan's law de Morgan's law de Morgan's law de Morgan's law distribution

23. $[p \wedge(q \vee r)] \rightarrow[(p \wedge q) \vee(p \wedge r)]$ distribution

24. $[p \vee(q \wedge r)] \Rightarrow[(p \vee q) \wedge(p \vee r)]$ distribution

25. $[(p \vee q) \wedge(p \vee r)] \rightarrow[p \vee(q \wedge r)]$ distribution

26. $[(p \wedge q) \vee(p \wedge \neg q)] \Rightarrow[p \wedge(q \vee \neg q)]$ distribution

27. $[(p \wedge q) \vee(p \wedge \neg q)] \Rightarrow p$

28. $[p \rightarrow(q \wedge r)] \Rightarrow[(p \rightarrow q) \wedge(p \rightarrow r)]$

29. $[(p \rightarrow q) \wedge(p \rightarrow r)] \rightarrow[p \rightarrow(q \wedge r)]$ 
30. $[(p \rightarrow r) \vee(q \rightarrow r)] \Rightarrow[(p \wedge q) \rightarrow r]$

31. $[(p \wedge q) \rightarrow r)] \rightarrow[(p \rightarrow r) \vee(q \rightarrow r)]$

32. $[p \rightarrow(q \rightarrow r)] \Rightarrow[(p \wedge q) \rightarrow r]$

33. $[(p \wedge q) \rightarrow r] \rightarrow[p \rightarrow(q \rightarrow r)]$

Notice that theses 23,25 , and 29 do not satisfy the reduction criterion $\mathrm{RD}$. The concept of strict validity in RMQ approximates very closely RC and RD (for details see [33]). This concept behaves in the same way as it can be seen by its theses with $\Rightarrow$.

Looking at de Morgan's laws 14-21 shows that strict validity in RMQ interprets a negation before parenthesis of a compound wff as weaker than the negation which is applied immediately to the wff. This coincides partially with intuitionistic logic, such that principles 18-21 hold only materially in RMQ (cf. [33, pp. 153f]).

Moreover, in the application to QM, the distribution laws hold only in one direction, namely from disjuncts to conjuncts (22 and 24). This is exactly what $\mathrm{RD}$ permits. $\mathrm{RD}$ rules out the other direction, invalid in the application to QM.

Finally, notice that also the following theses of CPC are strictly valid in RMQ:

34. $\neg(p \wedge \neg p)$

35. $p \vee \neg p$ principle of non-contradiction tertium non datur

\section{The Model RMQC of Causal Relations}

The logic of causal relations (or pluralistic causality) RMQC is an extension of the basic logic RMQ by adding the following five operations: $\mathrm{CF}, \mathrm{CN}, \mathrm{CS}_{1}, \mathrm{CS}_{2}$, and $\mathrm{CS}_{3}$.

\subsection{Definition of the system RMQC}

The system RMQC can be defined as the set of all formulas valid in the matrix $\mathfrak{M}_{\mathrm{RMQC}}$ of RMQC (or in the system RMQC). This matrix we obtain from the matrix $\mathfrak{M}_{\mathrm{RMQ}}$ from Section 5.1 (see pp. 476f.) and in which interpretations of additional operations $\mathrm{CF}, \mathrm{CN}, \mathrm{CS}_{1}, \mathrm{CS}_{2}, \mathrm{CS}_{3}$ are defined by the truth-tables from Table 5. By extending RMQ to RMQC all basic properties of the underlying logic RMQ are preserved. Thus, RMQC has all properties of RMQ listed in Section 5. 


\begin{tabular}{|c|llllll|}
\hline$p$ CF $q$ & 1 & 2 & 3 & 4 & 5 & 6 \\
\hline 1 & 6 & 1 & 2 & 5 & 5 & 6 \\
2 & 1 & 6 & 2 & 5 & 4 & 5 \\
3 & 1 & 4 & 6 & 5 & 4 & 3 \\
4 & 1 & 4 & 2 & 6 & 2 & 3 \\
5 & 1 & 1 & 3 & 3 & 6 & 3 \\
6 & 1 & 1 & 1 & 1 & 1 & 6 \\
\hline
\end{tabular}

\begin{tabular}{|c|llllll|}
\hline$p \mathrm{CN} q$ & 1 & 2 & 3 & 4 & 5 & 6 \\
\hline 1 & 6 & 1 & 3 & 1 & 3 & 1 \\
2 & 5 & 6 & 2 & 3 & 2 & 1 \\
3 & 5 & 4 & 6 & 3 & 2 & 5 \\
4 & 4 & 4 & 4 & 6 & 4 & 5 \\
5 & 5 & 5 & 5 & 5 & 6 & 5 \\
6 & 6 & 6 & 6 & 6 & 6 & 6 \\
\hline
\end{tabular}

\begin{tabular}{|c|c|c|}
\hline$p \mathrm{CS}_{1} q$ & 123456 \\
\hline 1 & 6165566 \\
2 & 1625556 \\
3 & 1565556 \\
4 & 1555656 \\
5 & 1233566 \\
6 & 666666 \\
\hline
\end{tabular}

\begin{tabular}{|c|l|l|}
\hline$p \mathrm{CS}_{2} q$ & 123456 \\
\hline 1 & 6565566 \\
2 & 165556 \\
3 & 156556 \\
4 & 1556556 \\
5 & 1235566 \\
6 & 6666666 \\
\hline
\end{tabular}

\begin{tabular}{|c|c|}
\hline$p \mathrm{CS}_{3} q$ & 123456 \\
\hline 1 & 62265666 \\
\hline 2 & 563566 \\
\hline 3 & 556566 \\
\hline 4 & 153666 \\
\hline 5 & 523566 \\
\hline 6 & 666666 \\
\hline
\end{tabular}

Table 5. The truth-tables for $\mathrm{CF}, \mathrm{CN}, \mathrm{CS}_{1}, \mathrm{CS}_{2}$, and $\mathrm{CS}_{3}$

In the subsequent sections, theses and non-theses of RMQC will be given. Since RMQC is decidable, so some important non-theses are listed, if they complement the understanding of the theses or of the respective causal relations.

\subsection{Theses of Basic Properties of $\mathrm{CF}, \mathrm{CN}, \mathrm{CS}_{1}, \mathrm{CS}_{2}$, and $\mathrm{CS}_{3}$}

Irreflexivity. For no causal relation it holds that a state of affairs or an event causes (or stands in causal relation to) itself. This is in agreement with the philosophical tradition (the only exception being Spinoza) and with modern physics: according to SR and GR every causal propagation needs time such that the cause must be earlier than the effect and nothing can be earlier than itself (recall Section 3.4 above).

It can be easily grasped from all truth-tables from Table 5 , by checking the value 6 (false) in the diagonal from top left to bottom right. Thus, for $C=\mathrm{CF}, \mathrm{CN}, \mathrm{CS}_{1}, \mathrm{CS}_{2}, \mathrm{CS}_{3}$ the formula (irr) from p. 469 is strictly valid in RMQC.

Transitivity. For $C:=\mathrm{CN}, \mathrm{CS}_{2}$ the formula (tr) from p. 469 is valid in RMQC, i.e.,

$$
\begin{aligned}
(p \mathrm{CN} q \wedge q \mathrm{CN} r) & \rightarrow p \mathrm{CN} r \\
\left(p \mathrm{CS}_{2} q \wedge q \mathrm{CS}_{2} r\right) & \rightarrow p \mathrm{CS}_{2} r
\end{aligned}
$$

are valid in RMQC. 
Not-transitivity. For $C:=\mathrm{CF}, \mathrm{CS}_{2}, \mathrm{CS}_{3}$ the formula (tr) is invalid in RMQC. So we can write (see the formula (not-tr) on p. 469):

$$
\begin{gathered}
(p \mathrm{CF} q \wedge q \mathrm{CF} r) \not \neg p \mathrm{CF} r \\
\left(p \mathrm{CS}_{1} q \wedge q \mathrm{CS}_{1} r\right) \not \neg p \mathrm{CS}_{1} r \\
\left(p \mathrm{CS}_{3} q \wedge q \mathrm{CS}_{3} r\right) \not \rightarrow p \mathrm{CS}_{3} r
\end{gathered}
$$

Whereas $\mathrm{CN}$ is usually transitive, CS is sometimes transitive and sometimes not. There are causal explanations, where CS is transitive (in the case of dynamical laws and Minkowski Space-Time), and others, where CS is not transitive (in the case of statistical laws and radiation processes). Moreover, to be a causal factor CF is not-transitive.

Asymmetry. For $C:=\mathrm{CN}, \mathrm{CS}_{2}, \mathrm{CS}_{3}$ the formula (asym) from p. 469 is valid in RMQC, i.e.,

$$
\begin{aligned}
& p \mathrm{CN} q \rightarrow \neg q \mathrm{CN} p \\
& p \mathrm{CS}_{2} q \rightarrow \neg q \mathrm{CS}_{2} p \\
& p \mathrm{CS}_{3} q \rightarrow \neg q \mathrm{CS}_{3} p
\end{aligned}
$$

are valid in RMQC. To prove (6.3) one needs first to turn the matrix on the diagonal top left-bottom right to receive $q \mathrm{CN} p$ from $p \mathrm{CN} q$ and then to exchange the six values with their negations to get $\neg q \mathrm{CN} p$. Finally, apply the truth-table of implication. Analogously for $\mathrm{CS}_{2}$ and $\mathrm{CS}_{3}$.

Asymmetry implies irreflexivity, and irreflexivity together with transitivity imply asymmetry. Accordingly, in RMQC those causal relations, which are irreflexive and transitive $\left(\mathrm{CN}, \mathrm{CS}_{2}\right)$ are also asymmetric. Moreover, $\mathrm{CS}_{3}$ is asymmetric, but not transitive. $\mathrm{CF}$ and $\mathrm{CS}_{1}$ are just not-symmetric.

Not-Symmetry. For $C:=\mathrm{CF}, \mathrm{CS}_{1}$ the formula (sym) is invalid in RMQC. So we can write (see the formula (not-sym) on p. 469):

$$
\begin{gathered}
p \mathrm{CF} q \not \rightarrow q \mathrm{CF} p \\
p \mathrm{CS}_{1} q \not \rightarrow q \mathrm{CS}_{1} p
\end{gathered}
$$

To prove (6.4) turn the matrix of $p$ CF $q$ like above and find some values of $p$ and of $q$ for which the implication is false.

Thus, there is a need for more concepts of sufficient cause, $\mathrm{CS}_{1}, \mathrm{CS}_{2}$, and $\mathrm{CS}_{3} . \mathrm{CS}_{2}$ is irreflexive, asymmetric, and transitive; $\mathrm{CS}_{1}$ is irreflexive, 
not-symmetric, and not-transitive; $\mathrm{CS}_{3}$ is irreflexive, asymmetric, and not-transitive.

Sufficient cause and sufficient condition. As we noted in Section 3.2, if $p$ is a sufficient cause for $q$, then $p$ is a sufficient condition for $q$, i.e., for all $\mathrm{CS}_{1}, \mathrm{CN}_{2}$, and $\mathrm{CN}_{3}$ the cases of the formula (3.1) are valid in RMQC:

$$
\begin{aligned}
& p \mathrm{CS}_{1} q \rightarrow(p \rightarrow q) \\
& p \mathrm{CS}_{2} q \rightarrow(p \rightarrow q) \\
& p \mathrm{CS}_{3} q \rightarrow(p \rightarrow q)
\end{aligned}
$$

Moreover, if $p$ is a sufficient cause for $q$, then $q$ is true, i.e., the following formulas are valid in RMQC:

$$
\begin{aligned}
& p \mathrm{CS}_{1} q \rightarrow q \\
& p \mathrm{CS}_{2} q \rightarrow q \\
& p \mathrm{CS}_{3} q \rightarrow q
\end{aligned}
$$

Necessary cause and necessary condition. As we noted in Section 3.2, if $p$ is a necessary cause for $q$, then $p$ is a necessary condition for $q$, i.e., the formula (3.2) is valid in RMQC. Moreover, if $p$ is a necessary cause for $q$, then $p$ is true, i.e., the the following formula is valid in RMQC:

$$
p \mathrm{CN} q \rightarrow p
$$

Logical closure. None of the five causal relations $\mathrm{CF}, \mathrm{CN}, \mathrm{CS}_{1}, \mathrm{CS}_{2}$, and $\mathrm{CS}_{3}$ satisfy logical closure in the following sense: If $p$ has one of the causal relations to $q$ and $q$ implies $r$, then $p$ has this causal relation to $r$. This, $C$ for any of five causal relations, is expressed in two versions by the following:

$$
\begin{aligned}
& (p C q \wedge(q \rightarrow r)) \not \supset p C r \\
& (p C q \wedge(q \Rightarrow r)) \not \supset p C r
\end{aligned}
$$

i.e., the corresponding material implications are not valid in RMQC. This is very important in the sense that they prevent taking logical consequences of states of affairs (even if they are causal effects) generally as causal effects too: logical consequence (logical derivation) does not preserve (transfer) causal relations.

\subsection{Interrelations among $\mathrm{CF}, \mathrm{CN}, \mathrm{CS}_{1}, \mathrm{CS}_{2}$, and $\mathrm{CS}_{3}$}

Causal Factor (CF). From Section 2.1 it is understandable that there are causal factors, which are more close to sufficient causes, and causal 
factors, which are close to necessary causes. The CF defined in Table 5 is a causal factor close to a sufficient cause. As will become clear from section 6.5, such a CF is needed in causal explanations as an initial condition together with the law as a necessary cause to cause sufficiently the effect. A CF as close to a necessary condition is not needed for the causal explanations given here and a respective definition is therefore dispensed with. Thus, CF as it is defined here (in Table 5) follows from sufficient cause:

$$
\begin{aligned}
& p \mathrm{CS}_{1} q \rightarrow p \mathrm{CF} q \\
& p \mathrm{CS}_{2} q \rightarrow p \mathrm{CF} q \\
& p \mathrm{CS}_{3} q \rightarrow p \mathrm{CF} q
\end{aligned}
$$

see (3.3) for $\mathrm{CS}:=\mathrm{CS}_{1}, \mathrm{CS}_{2}, \mathrm{CS}_{3}$.

For writing some theses of RMQC most accurately, it is suitable to use quantifiers for propositional variables. Since there is only a finite number of truth values, so $\forall p A(p)$ means: for all six values of $p$ we have $A(p)$, which is expressed by a conjunction over all six values: $A(1) \wedge$ $\cdots \wedge A(6)$. Similarly, $\exists p A(p)$ means: for some values of $p$ we have $A(p)$, which is expressed by a disjunction over six values of $p: A(1) \vee \cdots \vee A(6)$.

Independence among $\mathrm{CN}, \mathrm{CS}_{1}, \mathrm{CS}_{2}, \mathrm{CS}_{3}$. Instead of writing several theses by negating the respective universalized formulas, we abbreviate by:

None of any forms $\mathrm{CN}, \mathrm{CS}_{1}, \mathrm{CS}_{2}, \mathrm{CS}_{3}$ implies any of the others.

Connectedness as Common Cause. We say that a causal relation $C$ is connected in the sense of a common cause iff the following holds: For any two different states of affairs (events) $p$ and $q$, if neither $p$ causes $q$ nor $q$ causes $p$, then there is some other state of affairs (event) $r$ such that both $r$ causes $p$ and $r$ causes $q$.

This principle has also been called common cause principle and is usually attributed to Reichenbach [21]. The principle is very strong: The main question is whether one interprets the principle ontologically as holding of the causal order of nature or methodologically as a heuristic principle for the scientific search for causes or for explanations. In the latter case the principle should be rather formulated as a methodological norm: If of two different events $A$ and $B$ neither $A$ can be interpreted as a cause (reason, explanation) of $B$ nor $B$ for $A$, then search for a common cause for both $A$ and $B$. In this sense the principle is frequently used, 
both in every day life and in science. Interpreted ontologically, however, the principle seems to be too strong as a general principle, at least if causes are single events or states of affairs. In Minkowski Space-Time or in local regions of Riemannian Space-Time, an observer could in principle wait until such an event will appear in his backward light cone which causally can connect the two causally unrelated events. However, if gravitational fields and rotation of subsystems are included, the large scale structure does not allow such a possibility.

In a measurement process of QM, we cannot determine the result in the simple case, but for a large number of certain measurements of identically prepared systems, the relative frequency can be established. This reduced type of causality was called statistical causality by Wolfgang Pauli (cf. [14, pp. 32f.]). It is understandable that the common cause principle cannot be satisfied here.

The causal relations $\mathrm{CF}, \mathrm{CN}, \mathrm{CS}_{1}, \mathrm{CS}_{2}, \mathrm{CS}_{3}$ do not satisfy the principle of common cause or of connectedness. That means: If $C$ stands for any of five causal relations $\mathrm{CF}, \mathrm{CN}, \mathrm{CS}_{1}, \mathrm{CS}_{2}$, and $\mathrm{CS}_{3}$ (defined in Table 5), then the respective principle does not hold, i.e., we have:

$$
(\neg p C q \wedge \neg q C p) \not \rightarrow \exists r(r C p \wedge r C q)
$$

Moreover, it can be proved in RMQC that the common cause principle cannot be satisfied as a general principle of explanation in the sense that for any causally unrelated pair of events (states of affairs) there are initial conditions (as causal factors) and a connecting law as a necessary cause, where $C$ stands for one of five causal relations:

$$
(\neg p C q \wedge \neg q C p) \nrightarrow \neg[\exists r(r \mathrm{CF} p \wedge r \mathrm{CF} q) \wedge \exists r(r \mathrm{CN} p \wedge r \mathrm{CN} q)]
$$

This means: not for every pair of causally not related events $p$ and $q$ there is an explanatory event $r$ such that $r$ is a causal factor (initial condition) for both $p$ and $q$, and some other $r$ is a necessary cause for (stands in law-connection to) both $p$ and $q$.

The common cause principle cannot be defended either in the following weaker form as (where $C$ as above):

$$
(\neg p C q \wedge \neg q C p) \nrightarrow \neg \exists r(r \mathrm{CN} p \wedge r \mathrm{CN} q)
$$

This means: not for every pair of causally not related events $p$ and $q$ there is an event $r$ that has a law connection to both $p$ and $q$. 
However, the following weakenings for some states of affairs $p$ hold (where $C$ is either $\mathrm{CS}_{1}$, or $\mathrm{CS}_{2}$, or $\mathrm{CS}_{3}$, or $\mathrm{CN}$ ):

$$
\begin{aligned}
& \exists p \forall q[(\neg p C q \wedge \neg q C p) \rightarrow \exists r(r \mathrm{CN} p \wedge r \mathrm{CN} q)] \\
& \exists p \forall q[(\neg p C q \wedge \neg q C p) \rightarrow \exists r(r \mathrm{CF} p \wedge r \mathrm{CF} q)]
\end{aligned}
$$

If neither $p$ nor $q$ occur as states of affairs, then there cannot be a causal relation between them (where $C$ as above):

$$
(\neg p \wedge \neg q) \rightarrow(\neg p C q \wedge \neg q C p)
$$

Summing up: as a general principle, the common cause principle can only be defended as a heuristic methodological and normative rule. According to our model - as a general principle - it cannot be an ontological principle or a law-like statement about real causes. However, certain forms of the principle hold for some state of affairs $p$.

\subsection{Properties of $\mathrm{CN}, \mathrm{CS}_{2}, \mathrm{CS}_{3}, \mathrm{CS}_{1}, \mathrm{CF}$ satisfying causal sets}

The idea of the causal set is a hypothesis for the structure of space-time. It interprets the structure of space-time as a discrete causal order. The theory has been developed by Bombelli et al. [3], Sorkin [28], Reid [22], Markopoulou [16], Dowker [7], and others. In these theories, the causal order relation $C$ has three essential properties:

1. $C$ is transitive, i.e., for all $x, y, z:(x C y \wedge y C z) \rightarrow x C z$,

2. $C$ is non-circular, i.e., for all $x, y:(x C y \wedge y C x) \rightarrow x=y$,

3. $C$ is locally finite, i.e., every Alexandroff set $\{z: x C z \wedge z C y\}$ has only a finite number of elements (cf. [3, p. 522], [7, p. 1653]).

It can be shown that the causal relations $\mathrm{CN}$ and $\mathrm{CS}_{2}$ satisfy these three conditions completely, whereas $\mathrm{CS}_{1}, \mathrm{CS}_{3}$, and $\mathrm{CF}$ satisfies them partially.

Ad 1. $\mathrm{CN}$ and $\mathrm{CS}_{2}$ are transitive (cf. (6.1)).

Ad 2. Provided that states of affairs or events $x$ and $y$ (or $p$ and $q$ ) are different (which has been always assumed throughout this paper for $p$ and $q$ ) non-circularity is nothing else but asymmetry. Indeed, we obtain $x \neq y \rightarrow \neg(x C y \wedge y C x)$; so: $x \neq y \rightarrow(x C y \rightarrow \neg y C x)$.

By (6.3) we have: $\mathrm{CN}, \mathrm{CS}_{2}$, and $\mathrm{CS}_{3}$ are asymmetric and therefore they are also non-circular. $\mathrm{CS}_{1}$ and $\mathrm{CF}$ are not asymmetric, but at least not-symmetric and irreflexive.

Ad 3. We have to show first:

(i) For any $C$ of the five relations: $p C q$ allow intermediate states of affairs (events) $r$ such that $p C r \wedge r C q$ is compatible with $p C q$. 
Second, we want to show that:

(ii) The exclusion of such intermediate events, i.e., $\neg \exists r(p C r \wedge r C q)$, is compatible with $p C q$.

In this latter case $p C q$ is strictly discrete. An example for such two discrete events would be the two energy states of a quantum jump from the ground level to the first excited level.

Third, we want to postulate:

(iii) If there are intermediate states (events) as causes, then they are finite in number.

Ad (i) Compatibility with intermediate states as causes: Let $C$ be any of five causal relations $\mathrm{CN}, \mathrm{CS}_{1}, \mathrm{CS}_{2}, \mathrm{CS}_{3}$, and $\mathrm{CF}$. Then it holds:

$$
p C q \wedge \exists r(p C r \wedge r C q) \text { is compatible. }
$$

To prove (6.15), one has to check the matrix of the above conjunction. Since it contains both, values for true $(1,2,3)$ and values for false $(4,5$, 6 ) this conjunction is compatible. We may write therefore (6.15) also as follows:

$$
\exists p \exists q[p C q \wedge \exists r(p C r \wedge r C q)]
$$

This means that each of the five causal relations permits (is compatible with) intermediate causes (if there are any).

Ad (ii) Compatibility without intermediate states as causes: Let $C$ stand for any of five causal relations $\mathrm{CN}, \mathrm{CS}_{1}, \mathrm{CS}_{2}, \mathrm{CS}_{3}, \mathrm{CF}$. Then it holds:

$$
p C q \wedge \neg \exists r(p C r \wedge r C q) \text { is compatible. }
$$

This means that each of the five causal relations permits (is compatible with) no intermediate causes. In this case the Alexandroff set of $p C q$ is empty and $C$ is strongly discontinuous (discrete) (cf. Section 3.3). This can be proved in the same way as (6.15). It can also be formulated with the quantifiers $(\exists p \exists q)$.

Ad (iii) Locally finite: Since there is no restriction for a finite number of propositional variables (representing states of affairs or events) in RMQC we postulate that for any pair $\langle p, q\rangle$ of causally related events its Alexandroff set is at most finite.

Let $C$ stand for any of the five causal relations $\mathrm{CF}, \mathrm{CN}, \mathrm{CS}_{1}, \mathrm{CS}_{2}$, $\mathrm{CS}_{3}$, then the following postulate holds:

- If $p C q$ then the set $\{r: p C r \wedge r C q\}$ is at most finite. 


\subsection{Theorems of causal explanations}

We proceed here in the order of Section 4 with the different types of explanations.

6.5.1. Dynamical Laws. The state $S_{1}$ at time $t_{1}$ of the physical, chemical, biological or psychological system is a causal factor CF in the explanation. The dynamical law (or what it represents: the law-like structure or order of nature) is a necessary cause $\mathrm{CN}$. Both together are a sufficient cause for the effect, i.e., for the later state $S_{2}$ at time $t_{2}$. This is expressed by the following theses:

$$
\begin{gathered}
(p \mathrm{CF} q \wedge p \mathrm{CN} q) \mathrm{CS}_{2} q \\
p \mathrm{CF} q \wedge p \mathrm{CN} q \rightarrow p \wedge q
\end{gathered}
$$

Although time is not introduced here, that the cause must be earlier than the effect, is indirectly presupposed: Since no event can be earlier than itself, the causal relations must be irreflexive (see (irr)). Since the initial state $S_{1}\left(t_{1}\right)$ is earlier than the final state, the respective causal relation $\mathrm{CS}_{2}$ must be asymmetric (see (6.3)).

According to CL (Classical Mechanics) and SR (Special Relativity), the causal relation there (see [17, pp. 235ff]) is irreflexive, transitive, asymmetric, and continuous. The first three properties are satisfied also by $\mathrm{CN}$ and $\mathrm{CS}_{2}$ (see (irr), (6.1), and (6.3)). The condition of continuity is not satisfied, since the causality relations involved in this causal explanation, $\mathrm{CF}, \mathrm{CN}, \mathrm{CS}_{2}$, satisfy (CF partially) the conditions for causal sets (recall Section 6.4).

The thesis (6.18) says that if such a causal connection takes place then the occurrence of both the initial state and the final state are presupposed (implied). The thesis (6.18) together with (3.2), (6.5)-(6.7) should not be misunderstood. If any of the causal relations $\mathrm{CN}, \mathrm{CS}_{1}$, $\mathrm{CS}_{2}, \mathrm{CS}_{3}$ hold, then also the respective implications $q \rightarrow p$ or $p \rightarrow q$ hold. This is expressed (for the CS relations) by the following thesis (6.19), which follows from (6.5)-(6.10) (where CS stands for any of $\mathrm{CS}_{1}$, $\left.\mathrm{CS}_{2}, \mathrm{CS}_{3}\right)$ :

$$
\begin{aligned}
& (p \mathrm{CF} q \wedge p \mathrm{CN} q) \mathrm{CS} q \rightarrow[(p \mathrm{CF} q \wedge p \mathrm{CN} q) \rightarrow(p \rightarrow q)] \\
& (p \mathrm{CF} q \wedge p \mathrm{CN} q) \mathrm{CS} q \rightarrow[(p \mathrm{CF} q \wedge p \mathrm{CN} q) \rightarrow q]
\end{aligned}
$$

But the relations of implication are much weaker than the respective causal relations. An implication is a logical relation and from it, no 
causal relation follows. Thus, theorems (6.17), (6.20), and (6.21) are much stronger than (6.18) and (6.19). The three former theorems say that a certain relation of sufficient cause, with all its properties described in sections 6.2 and 6.4, holds between the two relata, not only an implication. This means that the values of the matrix of $p \mathrm{CF} q \wedge p \mathrm{CN} q$ are related to the values of $q$ in such a way that the matrix of the sufficient cause relation $\mathrm{CS}_{1}$, or $\mathrm{CS}_{2}$, or $\mathrm{CS}_{3}$ is satisfied.

For example, the value 1 of the matrix of $p \mathrm{CF} q \wedge p \mathrm{CN} q$ relates to the value 2 of $q$ by applying $\mathrm{CS}_{2}$ with result value 5 , whereas by applying $\mathrm{CS}_{1}$ with result the value 1 .

Observe moreover that (6.17), (6.20) and (6.21) are contingent, i.e., their matrices have both values true and values false; they are theses of RMQC. On the other hand, theses (irr), (3.2), (6.1)-(6.16), (6.18), and (6.19) of RMQC are not contingent in that sense, but logically true, i.e., their matrices contain only values for truth (i.e., the values from $\mathbf{T}:=\{1,2,3\})$. This means that RMQC is a factual theory and not just a system of some logic. We may say therefore that theses (irr), (3.2), (6.1)-(6.16), (6.18), and (6.19) are in a sense analytic relative to the system RMQC, whereas theorems (6.17), (6.20), and (6.21) are synthetic relative to RMQC.

6.5.2. Correlation Laws. As has been explained in Section 4.2, the General Gas Law $p \cdot V=R \cdot T$ can be considered on two levels: on the phenomenological and descriptive macro-level and on the micro-level underlying and producing the macro-level.

Macro-level: If we look for the suitable sufficient cause $\left(\mathrm{CS}_{1}, \mathrm{CS}_{2}\right.$, or $\mathrm{CS}_{3}$ ) for such an explanation, then we have to observe that this relation is not in general asymmetric, since a change of $V$ implies a change of $T$ and also a change of $T$ implies a change of $V$ (pressure being constant) and both changes are physically executable. Thus, we have to choose $\mathrm{CS}_{1}$, since only $\mathrm{CS}_{1}$ is not symmetric and not transitive. This choice is further supported because transitivity is not satisfied except in a trivial sense:

Both changes above, $C h(V)$ to $C h(T)$ and $C h(T)$ to $C h(V)$, lead by transitivity to $C h(V)$ to $C h(V)$ trivially. But for non-trivial cases, we would have to change also that magnitude, which is kept constant, and this destroys transitivity. Since $\mathrm{CS}_{1}$ is not symmetric and not transitive (see (6.4) and (6.2), respectively), it is the suitable causal relation here. 
On the other hand, the law as a necessary causal condition is always asymmetric and transitive.

$$
(p \mathrm{CF} q \wedge p \mathrm{CN} q) \mathrm{CS}_{1} q
$$

Micro-level: The situation is different on the micro-level. Here we have statistical laws not relevant for the singular case (for example: particle) but only for big ensembles. Since recurrence of a particular microstate is extremely improbable, we have to acquire asymmetry. Since a micro-state $m i$ is able to produce a macro-state $m a$ and this macro-state is able (with the law of entropy) to produce another macrostate (with higher entropy) $m a^{\prime}$ : the first micro-state $m i$ is also able to produce the latter macrostate $m a^{\prime}$. Higher entropy does not require that the original micro-state is different but only that the number of micro-states being able to realize $m a^{\prime}$ is greater than that realizing $m a$. Therefore transitivity is satisfied, and consequently, we have to choose $\mathrm{CS}_{2}$ which is both asymmetric and transitive: that means (6.17) holds also for the micro-level of Correlation Laws.

6.5.3. Law of Entropy. In causal explanations with the law of entropy, the entropy of the initial macrostate $m a$ at $t_{1}$ as a CF develops according to the law of entropy functioning as $\mathrm{CN}$ into the macrostate $m a^{\prime}$ at $t_{2}$ with higher entropy. Reversal of this process is extremely improbable, i.e., we have to require asymmetry for the sufficient cause. Furthermore, as has been shown in Section 4.3, transitivity is satisfied in the development of macro-states according to the law of entropy. Therefore, we have to choose $\mathrm{CS}_{2}$, which is asymmetric and transitive for the sufficient cause in such processes. Accordingly, (6.17) holds for such processes.

6.5.4. Quantum Jump. This case is different from the former: the sufficient cause cannot be transitive. An event $A$ (energy input by a photon) is capable to change electron's position (an event $B$ ) and $B$ is capable to emit a photon by jumping back (an event $C$ ); yet the event $A$ is not capable to produce the event $C$. The relation is also asymmetric: the energy input of the photon causes the electron to be on the excited state, but the excited state of the electron cannot cause an energy input of a photon. Therefore, we need a sufficient cause which is both asymmetric and not transitive: this is $\mathrm{CS}_{3}$. The respective explanation has the following form:

$$
(p \mathrm{CF} q \wedge p \mathrm{CN} q) \mathrm{CS}_{3} q
$$


6.5.5. Cloud Chamber. Two causal relations are involved here. The first is the decay-phenomenon which is caused by an electrostatic repulsion of the protons in heavy nuclei. This process is stochastic and is describable by statistical laws. Since in all such processes recurrence of the original state is extremely improbable, we have to require asymmetry. On the other hand, it does not seem possible to apply transitivity here in any reasonable sense. We may say that the electrostatic repulsion $(A)$ is able to produce the decay process $(B)$; but then, is $B$ able to produce the droplets $(C)$ ? It is rather the interaction of the charged particles with the atoms of the supersaturated vapour. And, certainly, $A$ is not able to produce $C$. Thus, we cannot assume transitivity. This means, we need a causal relation which is asymmetric but not transitive: this is $\mathrm{CS}_{3}$. Thus, for this first part of causal relation producing the $\alpha$-decay thesis (6.21) holds.

The second causal relation involved concerns the production of droplets due to the interaction of the charged particles with the atoms of the supersaturated vapour. Since laws of thermodynamics are involved and since on this (quantum-mechanical) level we have to interpret them on the micro-level recurrence is extremely improbable, such that asymmetry is required. On the other hand, similar to what has been said above concerning the first causal relation, transitivity does not seem to be applicable. Thus, the causal relation $\mathrm{CS}_{3}$ seems to be most suitable. Consequently, (6.21) holds also for the second part of causal relation involved in the experiment of the cloud chamber.

\subsection{Some Metaphysical Principles}

The following principles are theses of RMQC. We may call them "metaphysical principles", because they concern necessary and contingent states of affairs in general; specifically states of affairs that are either necessary or contingent but which are neither logically true nor logically false. The theorems state some important causal relations, implied by necessary or contingent states of affairs.

We use here the modal concepts $L$ and $M$ defined by the truth-tables from tables 1 and 3 and the Aristotelian concept of contingency $M p \wedge$ $M \neg p$ (i.e., possible $p$ and possible non- $p$ ) definable by the truth-tables from Table 6. Observe that this type of modal contingency is specific and different from the (weaker) contingency defined by a truth-table 


\begin{tabular}{|c|c|}
\hline$p$ & $M p \wedge M \neg p$ \\
\hline 1 & 6 \\
2 & 4 \\
3 & 1 \\
4 & 1 \\
5 & 4 \\
6 & 6 \\
\hline
\end{tabular}

Table 6. The truth-table for the Aristotelian concept of contingency

containing both, values for true and values for false, although $M p \wedge M \neg p$ satisfies this weaker condition too.

$$
\mathrm{L} p \rightarrow \exists q p \mathrm{CF} q
$$

Every necessary state of affairs is a causal factor for some state of affairs.

$$
\mathrm{L} p \rightarrow \exists q p \mathrm{CN} q
$$

Every necessary state of affairs is a necessary cause for some state of affairs.

$$
(\mathrm{M} p \wedge M \neg p) \rightarrow \exists q p \mathrm{CF} q
$$

Every contingent state of affairs is a causal factor for some state of affairs.

$$
(\mathrm{M} q \wedge \mathrm{M} \neg q) \rightarrow \exists p p \mathrm{CN} q
$$

Every contingent state of affairs has some necessary cause.

$$
(\mathrm{M} p \wedge \mathrm{M} \neg p) \rightarrow \exists q p \mathrm{CS}_{1} q
$$

Every contingent state of affairs is a sufficient cause $\mathrm{CS}_{1}$ for some state of affairs.

$$
(\mathrm{M} p \wedge \mathrm{M} \neg p) \rightarrow \exists q p \mathrm{CS}_{2} q
$$

Every contingent state of affairs is a sufficient cause $\mathrm{CS}_{2}$ for some state of affairs.

$$
(\mathrm{M} p \wedge M \neg p) \nrightarrow \neg \exists q p \mathrm{CS}_{3} q
$$

Not every contingent state of affairs is a sufficient cause $\mathrm{CS}_{3}$ for some state of affairs.

For any causal relation $C$ :

$$
M p \not \rightarrow \exists q p C q
$$

No causal relation to some states of affairs $q$ follows from a mere possibility (of $p$ ). 
Acknowledgments. The autor is indebted to an anonymous referee and to Professor Andrzej Pietruszczak for a number of valuable suggestions for transparency and improvement of an earlier version of the paper.

\section{References}

[1] Aristotle, Metayphysics. The Complete Works of Aristotle, Vol. 2, J. Barnes (ed.), Princeton, 1985.

[2] Barrow, J., and F. Tipler, The Anthropic Cosmological Principle, Oxford Univ. Press, Oxford, 1986.

[3] Bombelli, L., et al., "Space-time as a causal set", Physical Review Letters, 59 (1987): 521-524.

[4] Dell'Antonio, G.F., "On tracks in a cloud chamber", Foundations of Physics, 45, 1 (2015): 11-21. DOI: 10.1007/s10701-014-9850-9

[5] Dowe, P., "Wesley Salmon's process theory of causality and the conserved quantity theory", Philosophy of Science, 59, 2 (1992): 195-216. DOI: 10. $1086 / 289662$

[6] Dowe, P., Physical Causation. Cambridge Studies in Probability Induction and Decision Theory, Cambridge Univ. Press, Cambridge, 2000. DOI: 10. 1017/CB09780511570650

[7] Dowker, F., "Introduction to causal sets and their phenomenology", General Relativity and Gravitation, 45, 9 (2013): 1651-1667. DOI: 10.1007/ s10714-013-1569-y

[8] Gödel, K., "MAX PHIL X", transcribed by G. Crocco and E. M. Engelen.

[9] Hausman, D.M., Causal Asymmetries, Cambridge Univ. Press, Cambridge, 1998. DOI: $10.1017 /$ CBO9780511663710

[10] Hawking, S.W., and G.F.R. Ellis, The Large Scale Structure of Space Time, Cambridge Univ. Press, Cambridge, 1973. DOI: 10.1017/ CB09780511524646

[11] Hume, D., Enquiry into Human Understanding, L. A. Selby-Bigge (ed.), Oxford Univ. Press, 1902 (1961).

[12] Kant, I., Kritik der reinen Vernunft, Akademie Ausgabe, Berlin, 1905, vols. III and IV.

[13] Kiczuk, S., "The logic of causal propositions", Logic and Logical Philosophy 23, 4 (2014): 403-448. DOI: 10.12775/LLP. 2014.014

[14] Laurikainen, K.V., Beyond the Atom, Springer, Heidelberg, 1988. DOI: $10.1007 / 978-3-642-73852-4$

[15] Leibniz, G. W., Philosophische Schriften, C. I. Gerhardt (ed.), 7 volumes, Berlin, 1875-1890.

[16] Markopoulou, F., "Planck-scale models of the universe", chapter 24 in J. D. Barrow et al. (eds.), Science and Ultimate Reality, Cambridge Univ. Press, Cambridge, 2004. DOI: 10.1017/CB09780511814990.027 
[17] Mittelstaedt, P. and P. Weingartner, Laws of Nature, Springer, Heidelberg, 2005.

[18] Moffitt, T.E., et al., "Ein besseres Leben dank früher Selbstbeherrschung", in Spektrum der Wissenschaft, Dezember, 2014.

[19] Newton, I., Mathematical Principles of Natural Philosophy, F. Cajori (ed.), Univ. of California Press, Berkeley, 1934.

[20] Pearl, J., Causality. Models, Reasoning and Inference, Cambridge Univ. Press, Cambridge, 2000.

[21] Reichenbach, H., The Direction of Time, Univ. of California Press, Berkeley, 1956.

[22] Reid, D. D., "Introduction to causal sets: An alternate view of spacetime structure", 1999. http://arxiv.org/abs/gr-qc/9909075v1

[23] Salmon, W., Scientific Explanation and the Causal Structure of the World, Princeton Univ. Press, Princeton, 1984.

[24] Salmon, W., "Causality without counterfactuals", Philosophy of Science, 61 (1994): 297-312.

[25] Schurz, G., and P. Weingartner, "Versimilitude defined by relevant cosequence elements. A new reconstruction of Popper's original idea", pages 47-77 in Th. Kuipers (ed.) What is Closer-to-the-Truth?, Amsterdam, Rodopi, 1987.

[26] Schurz, G., and P. Weingartner, "Zwart and Franssen's impossibility theorem holds for possible world-accounts but not for consequence-accounts to versimilitude", Synthese, (2010): 415-436.

[27] Skyrms, B., Causal Necessity, Yale Univ. Press, New Haven and London, 1980.

[28] Sorkin, R. D., "First steps with causal sets", pages 68-90 in R. Cianci et al. (eds.), General Relativity and Gravitational Physics, World Scientific, Singapore, 1991.

[29] Thomas Aquinas, Summa Theologica, transl. by Fathers of the English Dominican Province, Christian Classics, Westminster, Maryland, 1948.

[30] Weingartner, P., Basic Questions on Truth, Kluver, Dordrecht, 2000. DOI: 10.1007/978-94-011-4034-8

[31] Weingartner, P., "Applications of logic outside logic and mathematics: Do such applications force us to deviate from classical logic?" pages 53-64 in W. Stelzner (ed.), Zwischen traditioneller und moderner Logik, Mentis, Paderborn, 2001.

[32] Weingartner, P., "Reasons from science for limiting classical logic", Chapter 15, pages 233-248, in P. Weingartner (ed.), Alternative Logics. Do Sciences Need Them?, Springer, Heidelberg, 2004. DOI: 10.1007/978-3662-05679-0_15

[33] Weingartner, P., "Matrix-based logic for application in physics", Review of Symbolic Logic 2, 1 (2009): 132-163. DOI: 10.1017/S1755020309090169 
[34] Weingartner, P., "A 6-valued calculus which avoids the paradoxes of deontic logic", in J.-Y. Beziau et al. (eds.), Conceptual Clarifications. Tributes to Patrick Suppes (1922-2014), College Publications, 2015.

[35] Weingartner, P., and G. Schurz (eds.), Law and Prediction in the Light of Chaos Research, vol. 473 of "Lecture Notes in Physics", Springer, BerlinHeidelberg, 1996. DOI: 10.1007/BFb0101863

[36] Woodward, J., Making Things Happen, Oxford Univ. Press, Oxford, 2003.

DOI: $10.1093 / 0195155270.001 .0001$

PAUl WeingarTner

Department of Philosophy

University of Salzburg

Franzsikanergasse 1

5020 Salzburg, Austria

paul. weingartner@sbg.ac at 